\title{
Is a clear benefit in survival enough to modify patient access to the surgery service? A retrospective analysis in a cohort of gastric cancer patients
}

\author{
Mattia Altini · Elisa Carretta • Paolo Morgagni · Tiziano Carradori • \\ Emanuele Ciotti · Elena Prati · Domenico Garcea • Amadori Dino • \\ Fabio Falcini • Oriana Nanni
}

Received: 19 September 2013/ Accepted: 13 January 2014/Published online: 30 January 2014

(c) The International Gastric Cancer Association and The Japanese Gastric Cancer Association 2014

\begin{abstract}
Background Surgery has become an important tool for cancer treatment, requiring many available resources and a good organization of the surgery service. The aim of this study was to provide robust data for policymakers on the impact of hospital volume on survival, taking into account different sources of information.

Methods We performed a retrospective study in a cohort of patients with gastric cancer submitted to partial or total gastrectomy. Data for the analysis were retrieved from regional administrative databases, the regional death registry, and histological reports. The main outcome measures were operative mortality and long-term survival. The associations between hospital volume and risk of mortality were calculated using a Cox multiple regression analysis. Results The estimated relationship between operative mortality and volume was not statistically significant. Conversely, high-volume hospitals had an increased
\end{abstract}

M. Altini $(\bowtie)$ - E. Carretta - E. Prati - A. Dino - F. Falcini · O. Nanni

Istituto Scientifico Romagnolo per lo Studio e la Cura dei

Tumori (IRST) IRCCS, Via Piero Maroncelli 40,

47014 Meldola, FC, Italy

e-mail: m.altini@irst.emr.it

P. Morgagni - D. Garcea

GB Morgagni General Hospital, Via Carlo Forlanini 34, 47121 Forlì, FC, Italy

T. Carradori

Directorate-General for Health and Social Policy,

Emilia Romagna Region, Viale Aldo Moro 21, 40127 Bologna, BO, Italy

E. Ciotti

Alma Mater Studiorum-University of Bologna, via sant'Isaia 90, 40123 Bologna, BO, Italy likelihood of long-term survival compared to low-volume institutions: hazard ratio 0.79 (95\% confidence interval, $0.66-0.94, p=0.01)$. The percentage variation between crude and adjusted HRs using only administrative data or administrative and histological data was very small. However, the combined use of administrative and clinical data provided a more accurate model for estimating riskadjusted mortality.

Conclusions A positive association between hospital volume and survival was evident for long-term outcome after adjusting for patient and tumor confounding. Moreover, the patient's choice of hospital was not guided by specific care pathways or screening programs, and prognosis was not poorer for patients in high-volume hospitals. These findings suggest that there is leeway for improving access to surgery for gastric cancer patients.

Keywords Gastric cancer · Gastrectomy - Survival · Hospital volume

\section{Introduction}

The Organisation for Economic Co-operation and Development (OECD) has stated that, since 2000, the Gross Domestic Product has grown an average of two percentage points less than average health spending per capita across OECD countries [1]. This fact highlights the need, today more than ever, for the good use of available resources, especially in national healthcare systems with a tradition of solidarity such as that of Italy. Within this context, clinicians and policymakers are under pressure to identify strategies to improve the quality and effectiveness of healthcare and, at the same time, to reduce costs.

Although surgery has become an important tool for cancer treatment, it also involves the significant use of 
resources. The relationship between volume and clinical outcome could be crucial to identifying evidence for planning improved organization of the surgery service. Numerous studies have examined the relationship between hospital volume and the clinical outcome of patients undergoing surgical procedures for cancer [2-16]. However, the volume-outcome relationship has not been always exploited to enhance the organization of the surgery service.

Our research focused on hospitals of Area Vasta Romagna (AVR), a catchment area located in the southeast of the Emilia Romagna region. The AVR Health Service is made up of four local Health Authorities with a total of 10 public hospitals and 11 private hospitals with a general surgery department. The area is characterized by a high incidence of gastric cancer: 16.5 (95\% CI 15.3-17.7) age-standardized incidence rate per 100,000 person-years among women and 32.8 (95\% CI 31-34.6) age-standardized incidence rate per 100,000 person-years among men (Romagna Cancer Registry, period 2004-2008; unpublished data).

The aim of the present study was to investigate the impact of hospital volume and other potential prognostic factors on the survival of patients undergoing gastric cancer surgery in AVR hospitals, taking into account different sources of information.

\section{Materials and methods}

We used regional administrative databases containing hospital discharge records (HDR) to identify consecutive patients submitted to surgery for gastric cancer from January 2004 to December 2008 in all the public and private hospitals located within AVR. To take into consideration a radical surgery approach, we restricted our sample to patients with a primary diagnosis code of stomach cancer $(151 . \times)$ and primary or secondary procedure codes of partial gastrectomy $(43.6,43.7,43.81,43.89)$ or total gastrectomy $(43.91,43.99)$, as defined by the International Classification of Diseases, 9th revision.

Patients who did not reside in the study area were excluded as their names would not have appeared in AVR death records. We linked the sample of patients with the Regional Death Registry and with histological referrals (only available for public hospitals). The Romagna Cancer Registry was also consulted to verify the diagnosis of stomach cancer and the outcome.

Access to data was granted by the Regional Health Authority and the Department of Healthcare Management of AVR hospitals. Data from the HDR database, the Regional Death Registry, and histological referrals were merged in a deterministic record-linkage procedure. The final database was anonymized by deleting the identity of the patients and other sensitive information and by assigning a unique numerical code to each individual. When anonymized administrative data are used for healthcare planning, studies are exempt from formal ethics review and specific written consent is not required to use patient information stored in hospital databases.

\section{Individual variables and hospital volume}

Details of demographic characteristics, information on coexisting medical conditions, and date and type of surgical procedure were obtained from hospital discharge records. The Charlson comorbidity index was used to evaluate the burden of comorbidities [17, 18].

Information on tumor stage, metastatic and surgically removed lymph nodes and lymph node stations, Lauren histology, and transected surgical margins was retrieved from histology referrals. The hospital volume of procedures was calculated as the mean annual number of gastrectomies performed per hospital. As no national standard volume has been established for gastrectomies in Italy and the study area is small, we considered the highest volume cutoff point used by several authors [2-4]. Thus, hospitals were classified into three volume categories: high-volume hospitals with more than 21 cases/year, low-volume hospitals with 7-21 cases/year, and very low volume hospitals with fewer than 7 cases/year.

\section{Outcome measures}

The patient outcomes of interest were operative mortality and long-term survival. Vital status was initially obtained from the Regional Death Registry and updated by the Romagna Cancer Registry. We defined operative mortality as time elapsed from surgery to death (all causes). If death occurred more than 30 days after the date of the surgical procedure or after hospital discharge, the patient was censored. Long-term survival was defined as time elapsed from the date of the surgical procedure to the date of death from any cause or to the date of the last follow-up. Followup data were available up to 31 December 2011. The hospital admission date was used when the date of the surgical procedure was missing for both operative mortality and long-term survival [5].

Statistical analysis

Patient and tumor characteristics from the three volume categories were compared using percentages and chisquare tests. The number of metastatic and removed lymph nodes was analyzed as both a ratio and separate variables [19]. 
We used the Kaplan-Meier method to estimate operative mortality and long-term survival between volume categories and the log-rank test to compare the curves. To identify potential prognostic factors associated with time-dependent clinical outcomes, we first developed a Cox model using a stepwise method and significance thresholds of 0.10 and 0.05 for covariate entry and removal, respectively. Removed stations were not taken into consideration in the multivariable analysis because this variable was not homogeneously reported by pathologists and also because we know that such data, retrieved from histological referrals, may reflect the different procedures of each hospital rather than actual disease conditions.

The adjusted hazard ratio for volume categories was assessed using a second Cox model comprising only the factors that proved statistically significant in the previous model. First-level interaction terms were included in regression models to test whether the impact of one variable on the outcome was modified by another variable. We checked for possible deviations from the proportional hazards assumption of the Cox model using the nonproportionality test on the basis of Schoenfeld residuals.

To reduce the potential impact of coding errors and identify the true radical treatment, we also implemented a sensitivity analysis by excluding patients with a secondary diagnosis of malignancy (197. $\times$ and 198. $\times$ ) and no specific indication of the surgical procedure performed, and patients whose tumors had infiltrated margins.

The amount of confounding from different sources of information was evaluated by the percentage variation between crude (from Cox regression model including only hospital volume as covariate) and adjusted HR for highvolume hospitals compared to low-volume hospitals in two nested models. The first model included only variables associated with outcomes retrieved from administrative data (model a), whereas the second added variables associated with outcomes from histological referrals to those retrieved from administrative data (model b). The very low category was not considered as we had no histological data for these hospitals. A percentage variation $\geq 10 \%$ between crude and adjusted HRs was considered as a relevant confounding contribution [20]. We compared the general goodness of fit of the nested models using the Akaike Information Criterion (AIC) [21] and the likelihood ratio test, assuming a chi-square distribution. A smaller AIC value indicated a more reliable model for predicting the outcome. The discriminative ability of the models was calculated using Herrell's c statistic [22]. The c statistic ranges from 0 to 1 , with higher values indicating better discrimination.
All tests were two-sided with a significance level of $<0.05$. Analyses were performed using SAS 9.3 (SAS Institute, Cary, NC, USA) and STATA 10 (StataCorp, College Station, TX, USA) software.

\section{Results}

During the 5-year study period, 1,435 patients with a diagnosis of stomach cancer underwent total or partial gastrectomy in the local area of interest. Of these, 121 $(8.4 \%)$ patients were excluded because they did not reside in the AVR area. A record linkage was obtained between administrative and clinical databases for patients discharged from public hospitals $(n=1,203 ; 91.6 \%)$. The patient sample underwent surgical treatment in 7 public hospitals and 9 private hospitals with a mean annual hospital volume ranging from 2 to 65 . Four public hospitals carried out high-volume surgery and three did low-volume surgery. All private hospitals were very low volume facilities.

Patient and tumor characteristics by hospital procedure volume are summarized in Table 1. Patients undergoing surgery in very low volume hospitals had a similar demographic profile but a lower prevalence of total gastrectomy procedures compared to low- and high-volume hospitals. Moreover, hospitals in the high-volume category showed a higher prevalence of removed lymph node stations and a different distribution of tumor stage compared to low-volume hospitals.

\section{Operative mortality}

Of the patients, $90(6.8 \%)$ died before being discharged from hospital or within 30 days of the surgical procedure. Overall, the operative mortality rate was $6.2 \%$ (95\% CI, 5.0-7.6\%), with great variability between hospitals. A minimum operative mortality rate of $4.23 \%$ was registered in a high-volume hospital and a maximum value of $11.58 \%$ was recorded in a low-volume hospital.

The Kaplan-Meier estimate of the operative mortality functions for the volume categories was not statistically significant $(p=0.16)$ (Fig. 1a).

In the multivariate analysis, the adjusted relationship between operative mortality and volume was not statistically significant (Table 2). Excluding patients who may not have been radically treated, the sensitivity test revealed weak evidence of a decreased risk of mortality for patients treated in high-volume hospitals compared to low-volume hospitals [HR, 0.56 (95\% CI, 0.31-1.00), $p=0.05]$. 
Table 1 Patient and tumor characteristics by hospital volume

\begin{tabular}{|c|c|c|c|c|}
\hline & \multicolumn{3}{|c|}{ Hospital volume } & \multirow[t]{2}{*}{$p$} \\
\hline & $\begin{array}{c}\text { Very low } \\
n=111 \\
\text { Number } \\
(\%)\end{array}$ & $\begin{array}{l}\text { Low } \\
n=278 \\
\text { Number } \\
\quad \%)\end{array}$ & $\begin{array}{l}\text { High } \\
n=925 \\
\text { Number }(\%)\end{array}$ & \\
\hline \multicolumn{5}{|c|}{ Data retrieved from HDR } \\
\hline \multicolumn{5}{|l|}{ Gender } \\
\hline M & $55(49.5)$ & $170(61.2)$ & $552(59.7)$ & \multirow{2}{*}{0.09} \\
\hline $\mathrm{F}$ & $56(50.5)$ & $108(38.8)$ & $373(40.3)$ & \\
\hline \multicolumn{5}{|l|}{ Age, years } \\
\hline$\leq 75$ & $60(54.1)$ & $150(54.0)$ & $521(56.3)$ & \multirow[t]{2}{*}{0.74} \\
\hline$>75$ & $51(45.9)$ & $128(46.0)$ & $404(43.7)$ & \\
\hline \multicolumn{5}{|l|}{ Charlson } \\
\hline 0 & 87 (78.4) & $221(79.5)$ & 719 (77.7) & \multirow[t]{3}{*}{0.91} \\
\hline 1 & $18(16.2)$ & 47 (16.9) & $162(17.5)$ & \\
\hline$\geq 2$ & $6(5.4)$ & $10(3.6)$ & $44(4.8)$ & \\
\hline \multicolumn{5}{|l|}{ Type of procedure } \\
\hline $\begin{array}{l}\text { Partial } \\
\text { gastrectomy }\end{array}$ & $85(76.58)$ & $164(58.99)$ & $482(52.11)$ & \multirow[t]{3}{*}{$<0.0001$} \\
\hline $\begin{array}{l}\text { Total } \\
\text { gastrectomy }\end{array}$ & $17(15.32)$ & $94(33.81)$ & $376(40.65)$ & \\
\hline Other & $9(8.11)$ & $20(7.19)$ & $67(7.24)$ & \\
\hline \multicolumn{5}{|c|}{ Data retrieved from HR } \\
\hline \multicolumn{5}{|c|}{ Metastatic lymph nodes ${ }^{\mathrm{a}}$} \\
\hline No & & $98(39.2)$ & $329(38.3)$ & \multirow[t]{6}{*}{0.87} \\
\hline N1 & & $36(14.4)$ & $140(16.3)$ & \\
\hline $\mathrm{N} 2$ & & $45(18.0)$ & $135(15.7)$ & \\
\hline $\mathrm{N} 3 \mathrm{a}$ & & $44(17.6)$ & $156(18.2)$ & \\
\hline $\mathrm{N} 3 \mathrm{~b}$ & & $27(10.8)$ & 99 (11.5) & \\
\hline Missing & & 28 & 66 & \\
\hline \multicolumn{5}{|l|}{ Removed station } \\
\hline$<6$ & & $238(94.8)$ & $580(67.8)$ & \multirow[t]{3}{*}{$<0.0001$} \\
\hline$\geq 6$ & & $13(5.2)$ & $276(32.2)$ & \\
\hline Missing & & 27 & 69 & \\
\hline \multicolumn{5}{|c|}{ Lymph nodes removed } \\
\hline$\leq 16$ & & $105(41.8)$ & $314(36.6)$ & \multirow[t]{3}{*}{0.13} \\
\hline$>16$ & & $146(58.2)$ & $544(63.4)$ & \\
\hline Missing & & 27 & 67 & \\
\hline \multicolumn{5}{|l|}{ Tumor stage } \\
\hline $\mathrm{T} 1$ & & $49(19.4)$ & $183(21.3)$ & \multirow[t]{5}{*}{0.006} \\
\hline $\mathrm{T} 2$ & & $30(11.9)$ & $132(15.4)$ & \\
\hline $\mathrm{T} 3$ & & $59(23.4)$ & $258(30)$ & \\
\hline $\mathrm{T} 4$ & & $114(45.2)$ & $287(33.4)$ & \\
\hline Missing & & 26 & 65 & \\
\hline \multicolumn{5}{|l|}{ Margin } \\
\hline Infiltrated & & $13(5.3)$ & $63(9.2)$ & \multirow[t]{3}{*}{0.06} \\
\hline Not infiltrated & & $231(94.7)$ & $625(90.8)$ & \\
\hline Missing & & 34 & 237 & \\
\hline \multicolumn{5}{|l|}{ Lauren } \\
\hline Intestinal & & $176(70.7)$ & $631(75.3)$ & 0.14 \\
\hline Diffuse/mixed & & $73(29.3)$ & $207(24.7)$ & \\
\hline Missing & & 29 & 87 & \\
\hline
\end{tabular}

Table 1 continued

\begin{tabular}{|c|c|c|c|c|}
\hline & \multicolumn{3}{|c|}{ Hospital volume } & \multirow[t]{2}{*}{$p$} \\
\hline & $\begin{array}{l}\text { Very low } \\
n=111 \\
\text { Number } \\
(\%)\end{array}$ & $\begin{array}{l}\text { Low } \\
n=278 \\
\text { Number } \\
(\%)\end{array}$ & $\begin{array}{l}\text { High } \\
n=925 \\
\text { Number (\%) }\end{array}$ & \\
\hline \multicolumn{5}{|l|}{ Ratio $^{\mathrm{b}}$} \\
\hline $\mathrm{N}$ ratio $0<15$ & & 44 (17.6) & 137 (16) & 0.64 \\
\hline $\mathrm{N}$ ratio $0 \geq 15$ & & $54(21.6)$ & $188(22)$ & \\
\hline $\mathrm{N}$ ratio 1 & & $17(6.8)$ & $85(9.9)$ & \\
\hline $\mathrm{N}$ ratio 2 & & $43(17.2)$ & $139(16.3)$ & \\
\hline $\mathrm{N}$ ratio 3 & & $92(36.8)$ & $306(35.8)$ & \\
\hline Missing & & 28 & 70 & \\
\hline
\end{tabular}

$H D R$ hospital discharge record, $H R$ histological report

${ }^{a} \mathrm{~N} 0=0$ positive lymph nodes; $\mathrm{N} 1=1-2$ positive lymph nodes; $\mathrm{N} 2=3-6$ positive lymph nodes, $\mathrm{N} 3 \mathrm{a}=7-15$ positive lymph nodes; $\mathrm{N} 3 \mathrm{~b} \geq 15$ positive lymph nodes

${ }^{\mathrm{b}} \mathrm{N}$ ratio $0<15=0 \%$ and removed lymph nodes $<15 ; \mathrm{N}$ ratio $0 \geq 15=0 \%$ and removed lymph nodes $\geq 15 ; \mathrm{N}$ ratio $1=1-9 \%$; ratio $2=10-25 \% ; \mathrm{N}$ ratio $3 \geq 25 \%[20]$

Long-term survival

There were 769 deaths (58.5\%) recorded and analyzed in relationship to long-term survival. The 3-year long-term survival rate was $51.2 \%(95 \% \mathrm{CI}, 48.5-53.9 \%)$ at a median follow-up of 69 months. Long-term survival across hospitals ranged from $87.5 \%$ to $39.3 \%$. However, excluding private hospitals, which represented a group of health institutions performing a lower number of surgical procedures and a higher percentage of partial gastrectomies, the range of variability between hospitals remained large (from $59 \%$ to $39 \%$ ).

Kaplan-Meier curves for the different volume categories showed a significant variation in long-term survival (high $51.9 \%$, low $45.7 \%$, very low $59.5 \%)(p=0.003)$ (Fig. 1b). After adjusting for the significant covariates, our results indicated an increased likelihood of long-term survival in patients undergoing surgical procedures in highvolume hospitals compared to low-volume hospitals (Table 2). The sensitivity analysis confirmed a benefit for high-volume hospitals compared to low-volume hospitals [HR, 0.77 (95\% CI, 0.63-0.95), $p=0.01]$.

\section{Accuracy of information sources}

The performance indicators of the two nested models are reported in Table 3. The HR percentage variation between crude and adjusted estimates was not significant for either model ( $a$ or b). However, model b had a lower AIC score for each outcome and a higher $c$-statistic to predict longterm survival. Moreover, the addition of tumor characteristics (model b) increased the likelihood ratio of operative 
Fig. 1 a Kaplan-Meier curves showing operative mortality among patients undergoing gastrectomy at very low, low-, and high-volume hospitals. b Kaplan-Meier curves showing long-term mortality in patients undergoing gastrectomy in very low, low-, and high-volume hospitals
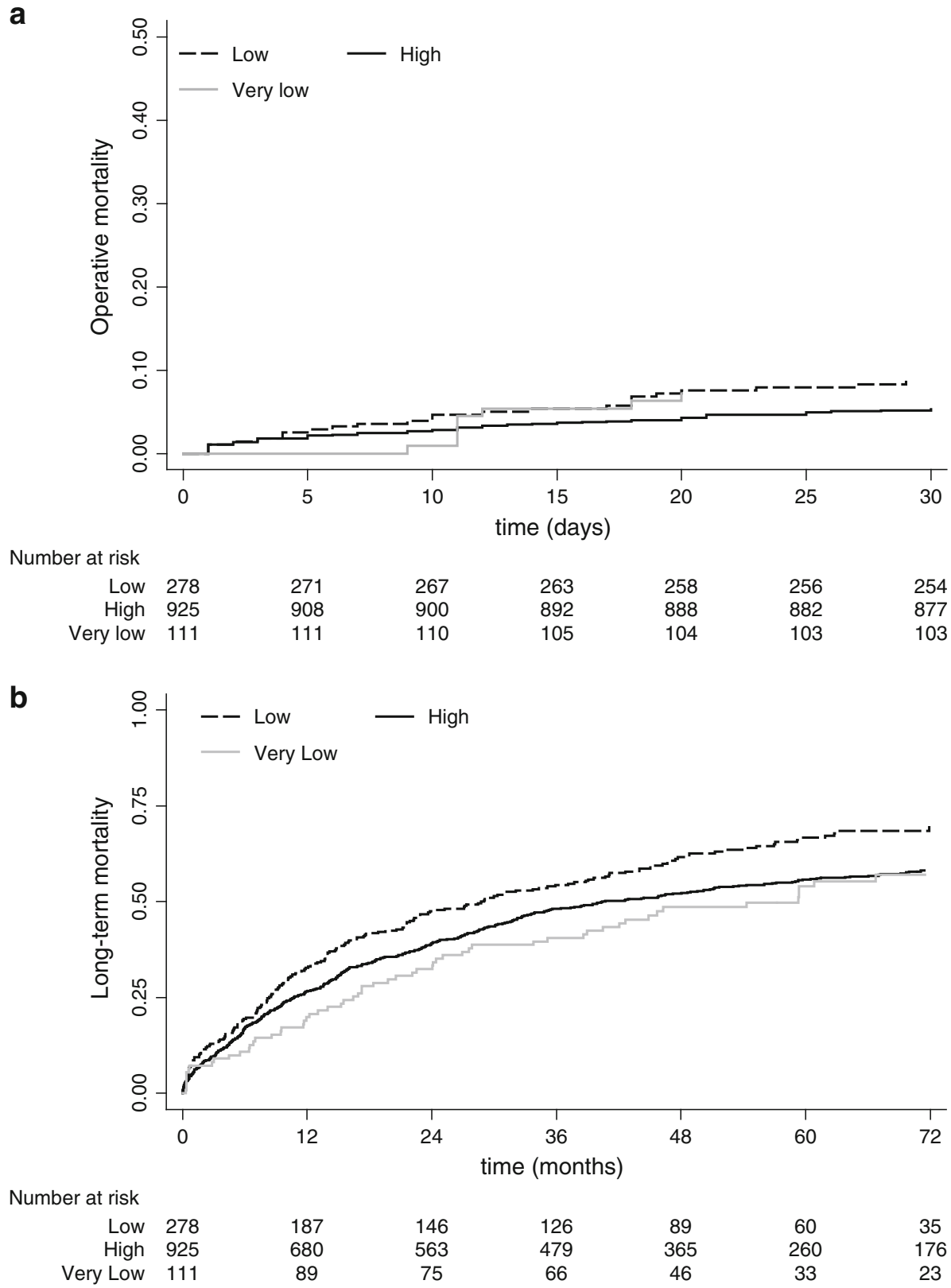

mortality by $23.7 \%(p<0.0001)$ and of long-term survival by $72.6 \%(p<0.001)$.

\section{Discussion}

Our study shows that patients who submitted to gastrectomies in high-volume hospitals had a $21 \%$ higher longterm survival than those undergoing the same procedure in low-volume hospitals. Weak evidence was found of a reduced risk of operative mortality for high-volume hospitals.

In the study area, the patient's choice of hospital was not guided by specific care pathways as there are no population-based screening programs associated with gastric cancer. We can thus exclude the possibility that patients with poor prognoses were concentrated in one specific hospital. The distribution of patient and tumour characteristics between high- and low-volume categories seems to confirm this assumption.

A limitation of our study is undoubtedly the restricted study population. However, patients living in the AVR area are characterized by epidemiological homogeneity, i.e., geographically stable populations with the same dietary habits, environmental conditions, and degree of exposure to exogenous agents.

The majority of published studies involve larger numbers of patients but use data retrieved solely from 
Table 2 Multivariate Cox models for operative and long-term survival

\begin{tabular}{|c|c|c|c|c|}
\hline & \multicolumn{2}{|c|}{ Operative mortality } & \multicolumn{2}{|c|}{ Long-term survival } \\
\hline & HR & $95 \% \mathrm{CI}$ & $\mathrm{HR}$ & $95 \% \mathrm{CI}$ \\
\hline \multicolumn{5}{|l|}{ Volume } \\
\hline Low & 1 & & 1 & \\
\hline High & 0.68 & $0.41-1.12$ & 0.79 & $0.66-0.94$ \\
\hline \multicolumn{5}{|l|}{ Gender } \\
\hline $\mathrm{F}$ & 1 & & 1 & \\
\hline M & 1.42 & $0.88-2.30$ & 1.21 & $1.03-1.43$ \\
\hline \multicolumn{5}{|c|}{ Age, years } \\
\hline$\leq 75$ & 1 & & 1 & \\
\hline$\geq 75$ & 3.80 & $2.24-6.43$ & 2.25 & $1.91-2.65$ \\
\hline \multicolumn{5}{|c|}{ Charlson } \\
\hline 0 & & & 1 & \\
\hline 1 & & & 1.31 & $1.07-1.60$ \\
\hline$\geq 2$ & & & 1.31 & $0.92-1.85$ \\
\hline \multicolumn{5}{|c|}{ Tumor stage } \\
\hline $\mathrm{T} 1$ & 1 & & 1 & \\
\hline $\mathrm{T} 2$ & 1.71 & $0.59-4.94$ & 1.28 & $0.90-1.82$ \\
\hline $\mathrm{T} 3$ & 2.23 & $0.89-5.55$ & 1.89 & $1.38-2.57$ \\
\hline $\mathrm{T} 4$ & 3.38 & $1.43-8.01$ & 2.85 & $2.09-3.88$ \\
\hline \multicolumn{5}{|c|}{ Type of procedure } \\
\hline Partial & & & 1 & \\
\hline Other & & & 0.83 & $0.58-1.20$ \\
\hline Total & & & 1.30 & $1.10-1.53$ \\
\hline \multicolumn{5}{|c|}{ Removed lymph nodes } \\
\hline$\geq 16$ & & & 1 & \\
\hline$\leq 16$ & & & 1.52 & $1.27-1.82$ \\
\hline \multicolumn{5}{|c|}{ Metastatic lymph nodes ${ }^{a}$} \\
\hline No & & & 1 & \\
\hline N1 & & & 1.54 & $1.18-2.02$ \\
\hline $\mathrm{N} 2$ & & & 2.19 & $1.69-2.84$ \\
\hline $\mathrm{N} 3 \mathrm{a}$ & & & 3.98 & $3.08-5.15$ \\
\hline $\mathrm{N} 3 \mathrm{~b}$ & & & 4.88 & $3.61-6.58$ \\
\hline
\end{tabular}

$H R$ hazard ratio

${ }^{\text {a }} \mathrm{N} 0=0$ positive lymph nodes; $\mathrm{N} 1=1-2$ positive lymph nodes; $\mathrm{N} 2=3-6$ positive lymph nodes; $\mathrm{N} 3 \mathrm{a}=7-15$ positive lymph nodes; $\mathrm{N} 3 \mathrm{~b} \geq 15$ positive lymph nodes

administrative databases leading to possible problems of poor-quality data and lack of completeness and accuracy $[6,7,23,24]$. The effort made in the present study to include linkage with histological referrals enabled us to assess the relationship between hospital volume and outcome after adjusting for tumor stage and removed and metastatic lymph nodes, which are known to be associated with outcome [25]. Furthermore, the combined use of administrative and clinical data from histological referrals provided the most accurate model for estimating the adjusted risks of death.
Currently, researchers approve the urge to improve the quality of cancer surgery. Volume could be defined as a proxy of the quality of care but other structural or process indicators may influence the relationship between volume and outcome. A recent study by Mayer and co-workers on radical cystectomy in England [5] reported an increased risk of mortality after adjustment for institutional structural and process variables. To our knowledge, few studies on gastrectomy include adjustment for these variables [11]. Unfortunately, we were unable to perform such an adjustment as information pertaining to these areas was not homogeneously recorded by the hospitals.

Furthermore, the analysis of surgeon volume could also be incorporated to explain outcome variations $[12,13]$. Surgeon volume, although probably highly correlated with hospital volume, may better reflect the effect of the surgical technique and of the expertise and skill of the surgical team on outcome.

The clinically appropriate cutoff used to define highvolume hospitals remains a controversial issue. A large number of different cutoff points have been used in previous studies, resulting in some authors defining a given volume of cases as "low" $[4,8]$ and others defining the same volume as "high" [7, 23]. Results from studies are thus often difficult to compare. We chose the only cutoff points used, to our knowledge, by more than one previous study to separate low-volume hospitals from medium- or high-volume hospitals [2-4]. We did not use conventional statistical cutoff points such as quartiles, which furnish approximately equal numbers of patients by volume categories. Interestingly, we observed a difference in the number of procedures carried out by private and public hospitals. In our population, private hospitals performed fewer than 7 gastrectomies/year, whereas all public hospitals carried out more than 18 gastrectomies/year. As we could only evaluate public hospitals at multivariate analysis, reducing the total number of hospitals assessed to 7, we decided to separate these hospitals into two groups. We opted to compare our results with those from other international studies rather than use a statistical cutoff that would increase heterogeneity and further limit the potential to generalize about the real effect of case volume on outcome.

In acknowledging these important limitations, our findings would appear to contradict those from the Dikken and co-workers [2] Netherlands study, which did not report a significant relationship between hospital volume and 6-month mortality or long-term survival. However, Dikken's work covered a period of two decades during which time diagnostic, preoperative staging, and patient care could have varied considerably. Moreover, only $2.5 \%$ of all gastrectomies were performed in high-volume Dutch hospitals. Conversely, the other two studies that used a cutoff of 21 procedures observed an association between 
Table 3 Accuracy indicators of HDR model (model a) and full model (model b)

\begin{tabular}{|c|c|c|c|c|}
\hline & \multicolumn{2}{|l|}{ Operative mortality } & \multicolumn{2}{|c|}{ Long-term survival } \\
\hline & Model a & Model b & Model a & Model b \\
\hline HR crude $(95 \% \mathrm{CI})$ & $0.64(0.40-1.01)$ & $0.64(0.40-1.01)$ & $0.77(0.65-0.91)$ & $0.77(0.65-0.91)$ \\
\hline HR adjusted (95\% CI) & $0.65(0.41-1.03)$ & $0.68(0.41-1.12)$ & $0.76(0.64-0.90)$ & $0.79(0.66-0.94)$ \\
\hline$\%$ HR variation ${ }^{\mathrm{a}}$ & $1.56 \%$ & $6.25 \%$ & $-1.30 \%$ & $2.47 \%$ \\
\hline $\mathrm{AIC}^{\mathrm{b}}$ & $1,122.84$ & 985.84 & $8,088.18$ & $8,040.22$ \\
\hline Likelihood ratio test $^{\mathrm{c}}$ & 48.67 & 60.22 & 146.79 & 536.53 \\
\hline$c$ Harrell concordance $(95 \% \mathrm{CI})^{\mathrm{d}}$ & $0.70(0.65-0.77)$ & $0.72(0.67-0.76)$ & $0.64(0.62-0.66)$ & $0.74(0.72-0.76)$ \\
\hline
\end{tabular}

Model a (HDR model) included only HDR variables associated with outcomes: volume, gender, and age for operative mortality and volume gender, age, Charlson index, and type of procedure for long-term survival

Model b (full model) included HDR and clinical variables associated with outcomes: volume, gender, age, and tumor stage for operative mortality and volume, gender, age, Charlson index, type of procedure, tumor stage, removed lymph nodes, and metastatic lymph nodes for longterm survival

$H D R$ hospital discharge record, $H R$ hazard ratio, AIC Akaike Information Criterion

${ }^{a}$ Higher percentage variation $(\geq 10 \%)$ indicates a model with relevant confounding information

${ }^{b}$ Smaller AIC value indicates a better model for predicting outcome

${ }^{c}$ Larger likelihood ratio test (chi-square value) indicates a model with improved fit

${ }^{\mathrm{d}}$ Higher $c$ value indicates a model with better discrimination of outcome

high-volume hospitals and low postoperative mortality for gastrectomy [3, 4].

A recent meta-analysis investigating the effect of hospital volume by cancer type revealed a significant association between volume and short- or long-term mortality in 14 of 20 studies on gastric cancer [26].

Considering the inverse volume-outcome relationship and other reasons, gastric cancer surgery has been centralized in some countries such as the UK, Denmark, The Netherlands, Sweden, and Finland [27]. Whether this approach is applicable to local condition and geography, there is a leeway for improving access to surgery for gastric cancer patients. In our region, in an effort to improve the management of resources and provide patients with standardized care pathways, the Regional Health Authority has plans to develop a comprehensive cancer network in which all services for cancer patients are centrally managed. Within this context, however, it is important to remember that surgeons must not be thought of as someone who performs surgical procedures throughout the network to facilitate patient access to a complete range of healthcare services. On the contrary, specific surgical expertise must be identified for single hospitals in the geographic area of interest on the basis of clinical outcomes obtained to ensure the best possible results for patients from the point of view of both medical technology and professional experience. At the same time, healthcare policy makers must be in a position to target the offer by taking into account evidence-based results to distinguish between low-complexity services that are available throughout the network and high-complexity services that are concentrated in reference centers.
In conclusion, in the area of oncological surgery, the robust data obtained in our study on the effect of hospital volume on survival strongly suggest that patients with gastric cancer should be referred to high-volume centers for surgery. Moreover, the information obtained in the present study encourage future research in other cancers and non-cancer pathologies for which surgery is one of the therapeutic elective choices.

Acknowledgments We are also grateful to the Pathology Units of the hospitals involved for providing the histological referrals used in the analysis, and to Ursula Elbling for editing the manuscript.

\section{References}

1. OECD. Health at a Glance 2011: OECD indicators. OECD Publishing. 2011. 10.1787/health_glance-2011-en.

2. Dikken JL, Dassen AE, Lemmens VE, et al. Effect of hospital volume on postoperative mortality and survival after oesophageal and gastric cancer surgery in the Netherlands between 1989 and 2009. Eur J Cancer. 2012;48:1004-13.

3. Birkmeyer JD, Siewers AE, Finlayson EV, et al. Hospital volume and surgical mortality in the United States. N Engl J Med. 2002;346:1128-9.

4. Anderson O, Ni Z, Møller H, et al. Hospital volume and survival in esophagectomy and gastrectomy for cancer. Eur $\mathrm{J}$ Cancer. 2011;47:2408-14.

5. Mayer EK, Bottle A, Darzi AW, et al. The volume-mortality relation for radical cystectomy in England: retrospective analysis of hospital episode statistics. BMJ. 2010;19(340):c1128.

6. Ghaferi AA, Birkmeyer JD, Dimick JB. Hospital volume and failure to rescue with high-risk surgery. Med Care. 2011;49: 1076-81.

7. Skipworth RJ, Parks RW, Stephens NA, et al. The relationship between hospital volume and post-operative mortality rates for 
upper gastrointestinal cancer resections: Scotland 1982-2003. Eur J Surg Oncol. 2010;36:141-7.

8. Finlayson EV, Goodney PP, Birkmeyer JD. Hospital volume and operative mortality in cancer surgery: a national study. Arch Surg. 2003;138:721-5.

9. Thompson AM, Rapson T, Gilbert FJ, et al. Scottish Audit of Gastric and Oesophageal Cancer. Hospital volume does not influence long-term survival of patients undergoing surgery for oesophageal or gastric cancer. Br J Surg. 2007;94:578-84.

10. Birkmeyer JD, Sun Y, Wong SL, et al. Hospital volume and late survival after cancer surgery. Ann Surg. 2007;245:777-83.

11. Smith DL, Elting LS, Learn PA, et al. Factors influencing the volume-outcome relationship in gastrectomies: a populationbased study. Ann Surg Oncol. 2007;14:1846-52.

12. Hannan EL, Radzyner M, Rubin D, et al. The influence of hospital and surgeon volume on in-hospital mortality for colectomy, gastrectomy, and lung lobectomy in patients with cancer. Surgery (St. Louis). 2002;131:6-15.

13. Derogar M, Sadr-Azodi O, Johar A, et al. Hospital and surgeon volume in relation to survival after esophageal cancer surgery in a population-based study. J Clin Oncol. 2013;31:551-7.

14. Meyerhardt JA, Tepper JE, Niedzwiecki D, et al. Impact of hospital procedure volume on surgical operation and long-term outcomes in high-risk curatively resected rectal cancer: findings from the Intergroup 0114 Study. J Clin Oncol. 2004;1(22):166-74.

15. Hu JC, Gold KF, Pashos CL, et al. Role of surgeon volume in radical prostatectomy outcomes. J Clin Oncol. 2003;1(21):401-5.

16. Hollenbeck BK, Dunn RL, Miller DC, et al. Volume-based referral for cancer surgery: informing the debate. J Clin Oncol. 2007;25:91-6.

17. Deyo RA, Cherkin DC, Ciol MA. Adapting a clinical comorbidity index for use with ICD-9-CM administrative databases. J Clin Epidemiol. 1992;45:613-9.
18. Quan H, Sundararajan V, Halfon P, et al. Coding algorithms for defining comorbidities in ICD-9-CM and ICD-10 administrative data. Med Care. 2005;43:1130-9.

19. Marchet A, Mocellin S, Ambrosi A, et al. The ratio between metastatic and examined lymph nodes ( $\mathrm{N}$ ratio) is an independent prognostic factor in gastric cancer regardless of the type of lymphadenectomy: results from an Italian multicentric study in 1853 patients. Ann Surg. 2007;245:543-52.

20. Rothman KJ. Epidemiology. An introduction. Oxford: Oxford University Press; 2002.

21. Akaike H. A new look at the statistical model identification. IEEE Trans Automat Contr AC. 1974;19:716-23.

22. Harrell FE Jr, Lee KL, Mark DB. Multivariable prognostic models: issues in developing models, evaluating assumptions and adequacy, and measuring and reducing errors. Stat Med. 1996;15:361-87.

23. Learn PA, Bach PB. A decade of mortality reductions in major oncologic surgery: the impact of centralization and quality improvement. Med Care. 2010;48:1041-9.

24. Fedeli U, Schievano E, Lisiero M. Mortality after esophageal and gastric cancer resection. World J Surg. 2012;36:2630-6.

25. Marrelli D, De Stefano A, de Manzoni G, et al. Prediction of recurrence after radical surgery for gastric cancer: a scoring system obtained from a prospective multicenter study. Ann Surg. 2005;241:247-55.

26. Gruen RL, Pitt V, Green S, et al. The effect of provider case volume on cancer mortality: systematic review and meta-analysis. CA Cancer J Clin. 2009;59:192-211.

27. Dikken JL, Verheij M, Cats A, Jansen EP, Hartgrink HH, van de Velde CJ. Extended lymph node dissection for gastric cancer from a European perspective. Gastric Cancer. 2011;14(4):396-8. 